\title{
Study of non-Newtonian polymer blends using large amplitude oscillatory shearing flow
}

\author{
Isameldeen E. Daffallah and Abdulwahab S. Almusallam* \\ Department of Chemical Engineering, Kuwait University, P.O. Box 5969, Safat 13060, Kuwait. \\ *Corresponding Author: Abdulwahab.almusallam@ku.edu.kw
}

Submitted :09/03/2021

Revised : 25/06/2021

Accepted :04/07/2021

\begin{abstract}
Large amplitude oscillatory shear (LAOS) was performed on non-Newtonian minor phase in Newtonian matrix phase polymer blends as a first step toward understating more complex immiscible polymer blends under high deformation condition. The blend consists of polybutadiene (PBD) as the droplet phase and polydimethylsiloxane (PDMS) as the matrix phase. The PBD droplet phase was an elastic "Boger" fluid prepared by dissolving a high-molecular-weight PBD into a low-molecular-weight Newtonian PBD. Different percentages of the high-molecular-weight PBD were used to prepare different types of Boger fluids that resulted in blends with different viscosity ratios from lower than unity, to unity, and higher than unity. Furthermore, the LAOS results of the blends were analyzed by using the Fourier Transform (FT) technique. From a theoretical point of view, the constrained volume model (CV-model) for Newtonian components is adapted to the case of a Newtonian matrix phase and non-Newtonian Boger fluid droplet phase by taking into account stresses that arise in the Boger fluids. The adapted model and the Newtonian CV-model were compared to the experimental results of FT-LAOS for checking the predictability of the model against the rheological properties. The adapted model shows some reasonable qualitative and quantitative agreements at high strain amplitude values.
\end{abstract}

Keywords: Constitutive equations; CV-model; Giesekus model; LAOS; Polymer blends.

\section{INTRODUCTION}

For immiscible polymer blends, the final mechanical properties are going to depend on the components that are used to make the blends and the interface that results from non-homogeneity (Verhulst, Moldenaers, and Minale, 2007). According to the science of rheology, the parent components of the immiscible polymer blend can be Newtonian or can be non-Newtonian fluids or a combination of both. The case of immiscible polymer blends composed of Newtonian polymers has been well studied experimentally, theoretically, numerically, and phenomenologically (Minale, 2010). The other cases such as the one where one phase is Newtonian and the other phase non-Newtonian, or the one where both of the two phases are non-Newtonian, are not well studied due to the complexity of the system (Minale, 2010).

The large amplitude oscillatory shear test (LAOS) is considered one of the most important rheological tests nowadays (K. Hyun et al., 2011) because it mimics the deformation conditions of manufacturing and processing. 
For analysing the LAOS tests, there are a lot of analysis techniques. The most famous technique is the Fourier transform (FT) and is considered as a fingerprint for studying the rheological response of complex materials $(\mathrm{K}$. Hyun et al., 2011).

From Hyun et al. (2011) literature review, we can see that LAOS technique is widely used to study the rheological properties of different systems such as entangled polymers, dispersion systems, and block copolymers and physical gels. For the specific case of immiscible polymer blends at LAOS conditions, some work has been carried out. Filipe et al. (2006) performed LAOS to study the linear and nonlinear rheological properties of liquid crystalline polymer (LCP) blended with polypropylene using a screw extruder. They concluded that the ratios of the third harmonic to the first harmonic obtained from analysing LAOS stresses by Fourier transform are able to describe the morphology of the blend along the extruder's screw better than the SAOS test. Grosso and Maffettone (2007) created a new technique for estimating the droplet size distribution of Newtonian droplets in a Newtonian matrix based on LAOS analyzed by Fourier transform. And they tested their new technique on synthetic data. Carotenuto et al. (2008) created a new technique to estimate droplet size and size distribution. The procedure is based on Fourier transform rheology, and they tested it by carrying out LAOS experiments on immiscible polymer blends of PDMS in PIB (a Newtonian liquid dispersed in a Newtonian matrix) and comparing the harmonics to those predicted by the Maffettone and Minale (MM) model. Also, they obtained a bimodal droplet distribution by fitting the real and imaginary parts of third and fifth harmonics. Moreover, they obtained volume-average droplet size for their blends that are consistently higher than those obtained using the Palierne model fit to the small amplitude oscillatory shear spectra (SAOS) of the same blend. Deyrail et al. (2009) studied two Newtonian liquidphase blends made of polyisobutylene and polydimethylsiloxane using different techniques such as optical microscopy, time resolved small angle light scattering (SALS), and linear-dichroism during LAOS flow. In addition, they investigated the prediction of Yu et al. (2002) model in terms of droplet deformation. Reinheimer et al. (2011) made a universal scaling law for the fifth harmonic peak over the third harmonic peak for different strain amplitudes and viscosity ratio values that are large enough to avoid large droplet deformation. Almusallam (2014) examined the CV model's predictions at LAOS conditions for immiscible blends composed of Newtonian components. At various viscosity ratio values, his model achieved good qualitative agreement with the experimental data for the third and fifth harmonics data. For the same case, a further study was done by Almusallam and Bini (2018) by comparing their experimental work with that of Jackson and Tucker. In addition, they developed a new scaling law based on Reinheimer et al. scaling law. Also, the LAOS technique is used to investigate the effect of compatibilizer on immiscible polymer blends and a lot of work has been done in this part by Hyun's group (Salehiyan, Choi, Lee, \& Hyun, 2014; Salehiyan \& Hyun, 2013; Salehiyan, Song, Choi, \& Hyun, 2015; Salehiyan, Song, Kim, Choi, \& Hyun, 2016; Salehiyan, Yoo, Choi, \& Hyun, 2014) and Ock et al., 2016.

From the literature review, it is apparent that there are no previous experimental studies that were carried out on LAOS in the case of immiscible non-Newtonian/Newtonian polymer blends. Besides, most of the phenomenological models that are available for immiscible blends are valid only for Newtonian components and small deformation (Jackson \& Tucker, 2003; Maffettone \& Minale, 1998; Peters, Hansen, \& Meijer, 2001; Yu \& Bousmina, 2003; Yu et al., 2002; and Yu \& Zhou, 2007), except a few models such as the CV-model, which was modified recently for large deformation (A.S. Almusallam, 2013). Also, there is no model tested under large deformation for non-Newtonian /Newtonian polymer blends.

Therefore, the main contribution of this work is to take an initial step toward understanding more complex non-Newtonian polymer blends experimentally and numerically under deformation that is close to manufacturing and processing conditions. And this could be achieved by firstly considering the case of one component to be a simple non-Newtonian polymer which is Boger fluid and the other component to be a Newtonian fluid, to mimic the high deformation that occurs in polymer manufacturing and processing by applying LOAS test and to use the most recent analysis technique FT-LAOS, which is the fingerprint for complex materials, to analyze the obtained 
results. And this could be the approach to help to understand the behaviour of various types of Non-Newtonian fluids blends such as the highly shear thinning character under this condition.

Based on that, the aims of this work can be summarized in three points. The first point is to obtain experimentally a general picture of LAOS in the case of non-Newtonian /Newtonian polymer blends. The second point is to modify the constrained-volume model for the case of non-Newtonian /Newtonian polymer blends. The final point is to compare the rheological experimental outcome to the modified constrained-volume model.

\section{LAOS BACKGROUND}

LAOS is performed in the non-linear viscoelastic region, which means it is performed at large strain amplitudes. Therefore, the relation between stress and the applied deformation is a non-linear relation. This nonlinear relation is quantified using different techniques such as Lissajous-Bowditch, the stress decomposition method, the orthogonal polynomial expansion method, and the Fourier transform method (K. Hyun et al., 2011). The most famous quantitative technique and also can be considered as a qualitative technique for analysing the stress signal is the Fourier transform (FT) technique (K. Hyun et al., 2011). In this method, the resultant stress signal at specified strain amplitude is collected as a function of time and then Fourier-transformed as follows:

$$
\begin{aligned}
& \sigma(t) \stackrel{F T}{\Leftrightarrow} \tilde{\sigma}(\omega) \\
& \tilde{\sigma}(\omega)=\int_{-\infty}^{+\infty} \sigma(t) e^{-i \omega t} \mathrm{dt}=\sum_{k=-\infty, o d d}^{k=\infty} c_{k} \delta\left(\omega-k \omega_{1}\right),
\end{aligned}
$$

where $\delta$ here is the Dirac delta function, $i$ is the imaginary number, $\omega_{1}$ is the characteristic angular frequency, and $c_{k}$ is the complex coefficient of the $k^{\text {th }}$ harmonic in the Fourier domain. And by taking the absolute value of the coefficient $c_{k}$, the intensity of the $k^{\text {th }}$ harmonic $\left(I_{k}\right)$ can be obtained.

\section{EXPERIMENTAL PROCEDURE}

\section{Materials Used}

For this work, polydimethylsiloxane (PDMS 30,000 g/mol, Sigma-Aldrich, USA) was used as a continuous phase. For the dispersed phase, a low molecular weight polybutadiene (PBD Mn 1,530 2,070g/mol , SigmaAldrich, Germany) was used and a high molecular weight polybutadiene (PBD 2x10 $\mathrm{g} / \mathrm{mol}$, Sigma-Aldrich, USA) was added as an adjustable component to prepare the different Boger fluids. Dichloromethane was used as a solvent for preparing the Boger fluids, which was purchased from CARLO ERBA Reagents, France.

\section{Preparation of Boger Fluids}

For the preparation of polybutadiene Boger fluids, an approach similar to that of Lerdwijitjarud et al. (Lerdwijitjarud, Larson, Sirivat, \& Solomon, 2003) was followed. First, the presence of volatile components in the pure polymer components was eliminated by placing the sample in a vacuum oven at $50{ }^{\circ} \mathrm{C}$ and down to $40 \mathrm{mbar}$. Secondly, the required quantity of the high molecular weight polybutadiene was measured and dissolved into dichloromethane. To ensure a proper dissolution, the mixture was placed into a bottle-rotator device at $50 \mathrm{rpm}$ for one day. After that, the low molecular weight PBD was added to the pre-dissolved high molecular weight PBD. Then, the mixture was left in the bottle-rotator device at $30 \mathrm{rpm}$ for one week. Finally, the present dichloromethane 
was eliminated from the mixture by placing the sample into the vacuum oven at $50{ }^{\circ} \mathrm{C}$ and down to 40 mbar until no weight loss was observed.

\section{Preparation of Blends}

Polydimethylsiloxane siloxane (PDMS), as matrix phase, was blended with Boger fluids at $20 \%$ volume fraction. To ensure the overall homogeneity of each blend, the components were mixed using a spatula for at least 15 minutes and until a uniform milky appearance was obtained. The blended samples were evacuated from entrained air by placing them in a desiccator for one night before the rheological measurements (A.S. Almusallam, 2014).

\section{Apparatus}

The rheology of the blend components was measured using ARES-G2, which is an air bearing rheometer, manufactured by TA Instruments (New Castle, DE, USA). The ARES-G2 rheometer has the ability to detect a minimum normal force equal to $0.001 \mathrm{~N}$ and a minimum torque equal to $0.05 \mu \mathrm{N}$.m. All the tests were performed at room temperature $\left(20^{\circ} \mathrm{C}\right)$ controlled by air condition $(\mathrm{AC})$.

\section{Loading Sample}

For loading the sample, $50 \mathrm{~mm}$ titanium cone and plate geometry with angle 0.0398 rad and with truncation gap equal to $0.0531 \mathrm{~mm}$ were used. The sample was trimmed at gap value of $0.008 \mathrm{~mm}$ to leave a slight bulge.

\section{CV-MODEL BACKGROUND}

In 1991, Doi and Ohta (Doi \& Ohta, 1991) developed a theory that predicts the rheology of co-continuous morphology of two Newtonian phase blends with a unity viscosity ratio. Based on this theory, Almusallam et al. developed a model, called the constrained volume model (CV-model), for droplet morphologyy composed of Newtonian constituents where the viscosity ratio between constituents can be less than unity, equal to unity, and higher than unity. For the CV model, the anisotropy tensor $\left(\hat{q}_{\alpha \beta}\right)$ is represented in similar way to Doi and Ohta theory:

$$
\hat{q}_{\alpha \beta}=\frac{1}{V_{d}} \int_{S_{d}}\left(n_{\alpha} n_{\beta}\right) d S_{d}
$$

where $V_{d}$ and $S_{d}$ represent the volume and the interface of the droplet, respectively. $n_{\alpha}\left(n_{\beta}\right)$ denotes the alpha (beta) component of a unit vector normal to the interface as can be seen in Fig. 1. A full description of the model can be found in a previous work for Almusallam (A.S. Almusallam, 2013). 

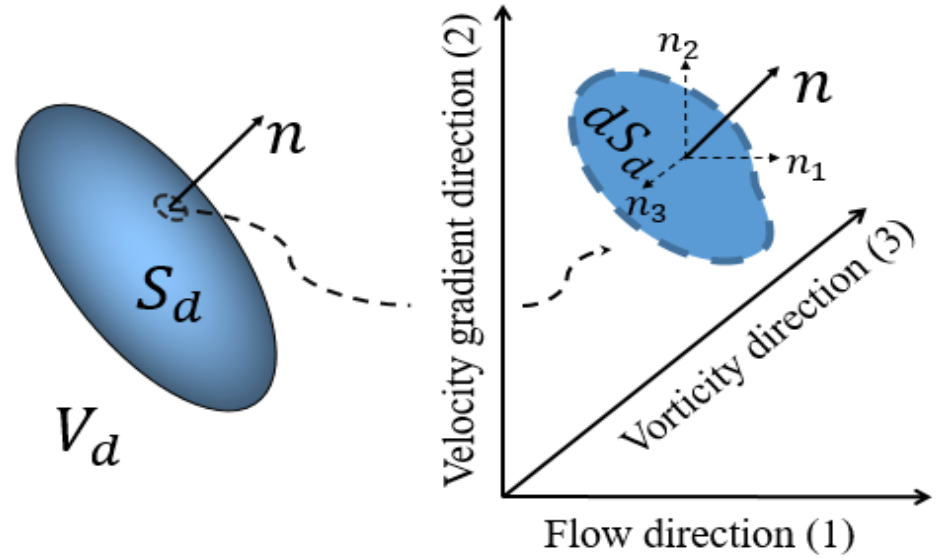

Figure 1. Illustration diagram showing the volume of a deformed droplet, the surface area, and a unit vector normal to the interface and its orthogonal components, where the vorticity direction is pointing toward the reader.

\section{CV-MODEL FOR NON-NEWTONIAN DROPLETS}

The CV-model is modified to account for the presence of a non-Newtonian droplet phase by changing the way of calculating the viscosity of the matrix phase and the droplet phase, which is done by creating a representative viscosity that accounts for the Boger fluid behavior (as manifested in the presence of normal stresses).

The droplet representative viscosity $\left(\eta_{\exists}^{*}\right)$ is a sum of a weighted average of the shear viscosity and the extensional viscosity:

$$
\eta_{\exists}^{*}=\left(1-\lambda_{M z}\right) \eta_{\text {shear }}^{*}+\lambda_{M z} \eta_{\text {extentional }}^{*}
$$

The $\eta_{\text {shear }}^{*}$ is simply equal to the droplet viscosity $\eta_{d} . \lambda_{M z}$ is a parameter that helps to weight the contributions of the shearing and extensional viscosities. For weighting of the viscosity contributions, Cheng and Manas-Zloczower technique (Cheng \& Manas-Zloczower, 1990) is used. The parameter $\lambda_{M z}$ can be described in the following equation:

$$
\lambda_{M Z}=\frac{\sqrt{D_{\alpha \beta}^{*}: D_{\alpha \beta}^{*}}}{\sqrt{D_{\alpha \beta}^{*}: D_{\alpha \beta}^{*}}+\sqrt{\Omega_{\alpha \beta}^{*}: \Omega_{\alpha \beta}^{*}}},
$$

where $\Omega_{\alpha \beta}^{*}$ is the imposed vorticity tensor in the droplet phase and $D_{\alpha \beta}^{*}$ is the imposed rate of deformation tensor in the droplet phase. The advantage of Cheng and Manas-Zloczower technique is that the contributions of shearing, extensional, and pure rotational flows are weighted using the deformation and the vorticity rates. Also, the weighting function ranges from [0-1] and the ratio $\left(\lambda_{M Z}\right)$ is equals to 1 for the case of purely extensional flow, 0.5 for purely shearing, and 0 for purely rotational flow. 
The extensional viscosity is assumed to be that of an Oldroyd-B fluid, which according to Bird and Hassager (1987) can be defined from the Giesekus model as follows:

$$
\begin{aligned}
& \eta_{\text {extentional }}^{*}=3 \eta_{o}^{*} *\left\{\frac{\lambda_{2}}{\lambda_{1}}+\left(1-\frac{\lambda_{2}}{\lambda_{1}}\right) \frac{1}{6 \alpha_{G}}\left[3+\frac{1}{\lambda_{1} \dot{\epsilon}^{*}} * \mathcal{H}\right]\right\}, \\
& \mathcal{H}=\left\{\left[1-4\left(1-2 \alpha_{G}\right) \lambda_{1} \dot{\epsilon}^{*}+4 \lambda_{1}^{2} \dot{\epsilon}^{* 2}\right]^{\frac{1}{2}}-\left[1+2\left(1-2 \alpha_{G}\right) \lambda_{1} \dot{\epsilon}^{*}+\lambda_{1}^{2} \dot{\epsilon}^{* 2}\right]^{\frac{1}{2}}\right\},
\end{aligned}
$$

where $\lambda_{1}$ and $\lambda_{2}$ are relaxation and retardation times of the non-Newtonian fluid, respectively. And $\alpha_{G}$ is the mobility factor for Giesekus constitutive equation.

The extension rate inside the droplet is approximated as follows (Debbaut \& Crochet, 1988):

$$
\dot{\varepsilon}=6 \mathrm{III}_{D_{\alpha \beta}^{*}} / \mathrm{II}_{D_{\alpha \beta}^{*}},
$$

where $\mathrm{II}_{D_{\alpha \beta}^{*}}$ and $\mathrm{III}_{D_{\alpha \beta}^{*}}$ are the second and third invariants of the anisotropy tensor.

For calculating the viscosity ratio $\eta_{r}$, the continuity of the droplet expansion to the matrix phase needs to be enforced, which means that as the droplet expands, the matrix phase also expands. Therefore, the matrix viscosity is defined in a similar way to that used to model the droplet phase:

$$
\eta_{\exists}^{m}=\left(1-\lambda_{M z}\right) \eta_{\text {shear }}+\lambda_{M z} \eta_{\text {extentional }}
$$

The Cheng and Manas-Zloczower factor $\left(\lambda_{M z}\right)$ is that of the droplet phase, $\eta_{\text {shear }}$ is equal to the matrix viscosity $\eta_{m}$. Furthermore, we set $\eta_{\text {extentional }}$ for the matrix phase to be $3 \eta_{\text {shear }}$ so that the viscosity ratio between the droplet phase and the matrix phase is consistent with the Newtonian/Newtonian limit. The model viscosity ratio is now modified to $\eta_{r}=\eta_{\exists}^{*} / \eta_{\exists}^{m}$.

\section{CONSTITUTIVE EQUATIONS}

For stress calculations, the Aggarwal and Sarkar (2007) technique was used for representing the constitutive equation of emulsions, which can be represented as follows:

$$
\sigma_{\alpha \beta}^{\text {Total }}=2 \eta_{m} D_{\alpha \beta}+\sigma_{\alpha \beta}^{\text {Minor }}-p \delta_{\alpha \beta}
$$

In our work, the major phase is Newtonian, and therefore it can be directly represented by Newton's law of viscosity as can be seen in the first term of Eq. 9. The last term in Eq. 9 is the isotropic stress, where $p$ is the isotropic pressure and $\delta_{\alpha \beta}$ is the unit tensor.

The minor phase is non-Newtonian, and therefore the stress in the minor phase $\left(\sigma_{\alpha \beta}{ }^{\text {Minor }}\right)$ is presented by a sum of elastic, viscous, and interfacial contributions: 


$$
\sigma_{\alpha \beta}^{\text {Minor }}=\sigma_{\alpha \beta}^{\text {elastic }}+\sigma_{\alpha \beta}^{\text {viscous }}+\sigma_{\alpha \beta}^{\text {Interface }} \text {. }
$$

The purely elastic stress contribution of the droplet phase can be calculated by using the following equation:

$$
\sigma_{\alpha \beta}^{\text {elastic }}=\phi\left(\sigma_{d, \alpha \beta}-2 \eta_{d} D_{\alpha \beta}^{*}\right) \text {. }
$$

For calculating the total viscoelastic stress of the droplet $\left(\sigma_{d, \alpha \beta}\right)$, Giesekus constitutive equation is used. Giesekus constitutive equation can be represented as follows (Bird \& Hassager, 1987):

$$
\begin{aligned}
& \sigma_{d, \alpha \beta}=2 \eta_{s d} D_{\alpha \beta}^{*}+\sigma_{p d, \alpha \beta}, \\
& \sigma_{p d, \alpha \beta}+\lambda_{1}\left[\sigma_{p d, \alpha \beta}\right]+\alpha_{G} \frac{\lambda_{1}}{\eta_{p d}}\left\{\sigma_{p d, \alpha \beta} \cdot \sigma_{p d, \alpha \beta}\right\}=2 \eta_{p d} D_{\alpha \beta}^{*} .
\end{aligned}
$$

Giesekus constitutive equation is composed of two parts. The first part in Eq. 12 is the Newtonian solvent stress which is in our work the low molecular weight PBD. And the second part is the non-Newtonian polymeric stress $\left(\sigma_{p d, \alpha \beta}\right)$. Moreover, $\eta_{s d}$ and $\eta_{p d}$ are the the solvent and polymer contributions to viscosity, and $\alpha_{G}$ is Giesekus mobility factor.

The stress that results from the mismatch between the major and minor phase viscosities can be represented from Yu and Bousmina (2003) as follows:

$$
\sigma_{\alpha \beta}^{\text {viscous }}=2\left(\eta_{d}-\eta_{m}\right) \phi D_{\alpha \beta}^{*}
$$

For interface stress calculations, Yu and Bousmina formula (Yu \& Bousmina, 2003) was used:

$$
\sigma_{\alpha \beta}^{\text {Interface }}=-\Gamma \phi\left(\widehat{q}_{\alpha \beta}-1 / 3 I_{\widehat{q}} \delta_{\alpha \beta}\right)
$$

where $\Gamma$ is the interfacial tension $\operatorname{and} I_{\hat{q}}$ is the first invariant of the anisotropy tensor.

\section{RESULTS AND DISCUSSION}

\section{Characterization of Pure Components and Boger Fluids}

In Fig.2(a) the viscosities of the pure PBD and pure PDMS can be seen to be independent of shear rate. For the $5 \%$ Boger fluid, the viscosity is almost independent of shear rate in the range of the test from 0.01 to $10 \mathrm{~s}^{-1}$ and a weak shear thinning behaviour after that. On the other hand, for $7.5 \%$ Boger fluid, the viscosity is constant up to a shear rate equal to $2 \mathrm{~s}^{-1}$. After this point, it shows a weak shear thinning, while the $10 \%$ Boger fluid shows strong shear thinning after $2 \mathrm{~s}^{-1}$. Furthermore, increasing the amount of the high molecular weight PBD in preparing the 
Boger fluids resulted in increasing the viscosity. The zero-shear viscosity for the pure components and Boger fluids are summarized in Table 1.

In Fig. 2(b), the presence of the first normal stress difference can be observed in all of the Boger fluids that are going to be used to form the minor phase for the model polymer blends. The values of first normal stress difference for the PDMS are less than the 5.0\% Boger fluid, while they are orders of magnitude less than those for the other Boger fluids. For the pure PBD, no first normal stress difference was detected.

In Fig. 2, the fitting quality of determining the mobility factor of Giesekus constitutive equation for the prepared Boger fluids is presented. The mobility factor was obtained by fitting the experimental viscosity and the first normal stress difference coefficient with the steady-state predictions of the Giesekus constitutive equation. The values obtained from the fits are summarized in Table1.

Table 1. The zero-shear viscosity for the pure components and Boger fluids, the relaxation time, Giesekus mobility factor, and the solvent and polymer contributions to viscosity are obtained by fitting the experimental data to the steady state predictions of Giesekus model.

\begin{tabular}{|l|c|c|c|c|c|}
\hline Type of the sample & $\boldsymbol{\eta}_{\boldsymbol{o}}($ Pa.s) & $\lambda_{\mathbf{1}}(\mathbf{s})$ & $\boldsymbol{\alpha}_{\boldsymbol{G}}$ & $\boldsymbol{\eta}_{\boldsymbol{s} \boldsymbol{d}}$ (Pa.s) & $\boldsymbol{\eta}_{\boldsymbol{p d}}$ (Pa.s) \\
\hline Pure PBD & 0.816 & - & - & - & - \\
\hline Pure PDMS & 32.350 & - & - & - & - \\
\hline $5.0 \%$ Boger & 10.030 & 0.0356 & 0.3810 & 3.295 & 6.735 \\
\hline $7.5 \%$ Boger & 29.639 & 0.0413 & 0.9945 & 6.972 & 22.667 \\
\hline $10 \%$ Boger & 199.637 & 0.1820 & 0.9210 & 24.755 & 174.882 \\
\hline
\end{tabular}


(a)

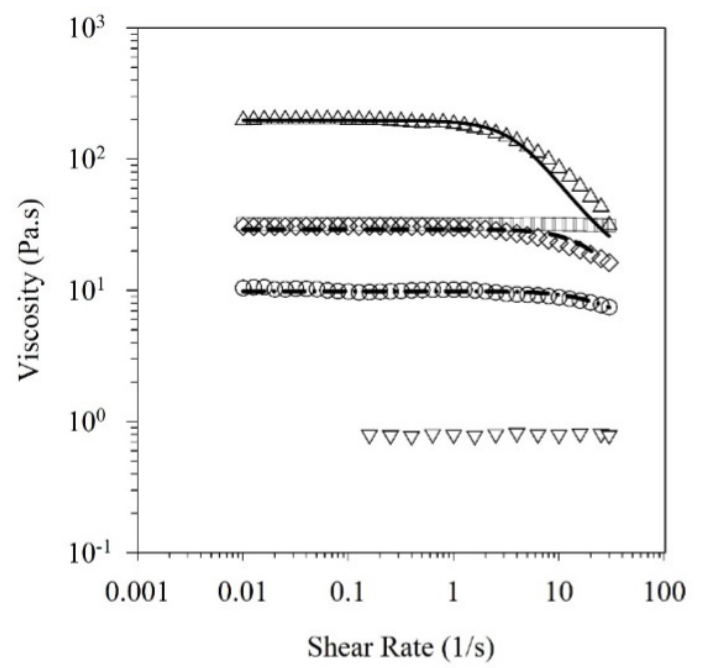

(c)

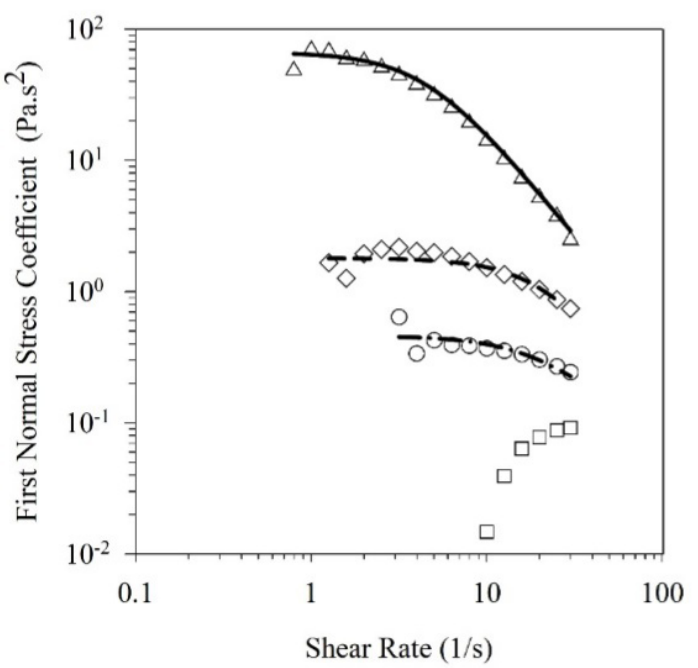

(b)

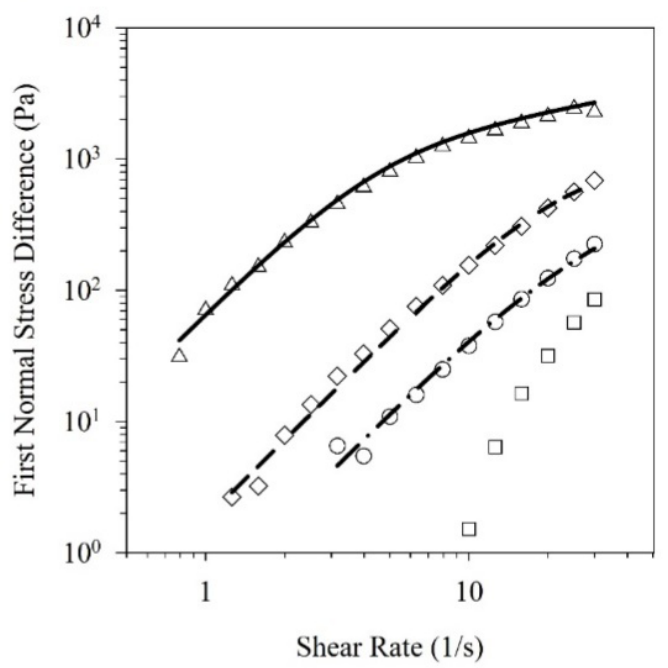

Shear Rate $(1 / \mathrm{s})$

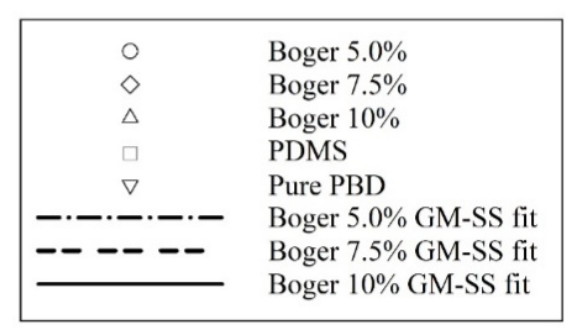

Figure 2. The experimental flow sweep test and the steady-state Giesekus model fit (GM-SS):

(a) the viscosity versus the shear rate for pure components and Boger fluids,

(b) the first normal stress difference values, and

(c) the first normal stress coefficient values. 
(a)

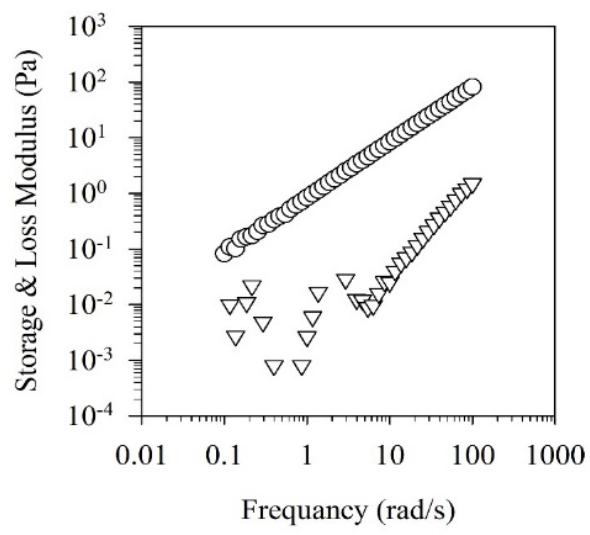

(c)

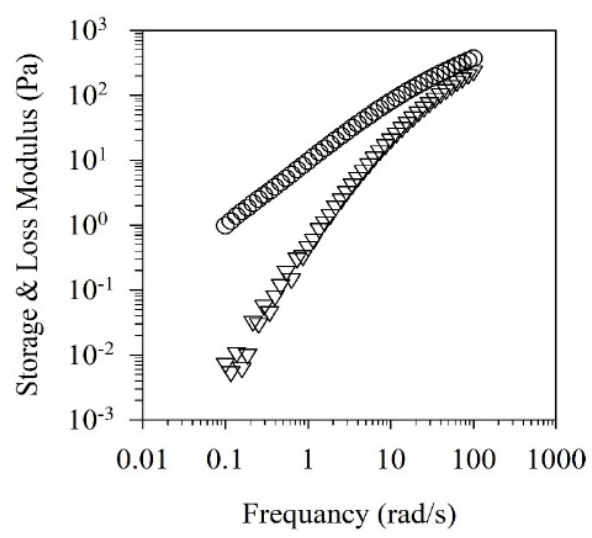

(e)

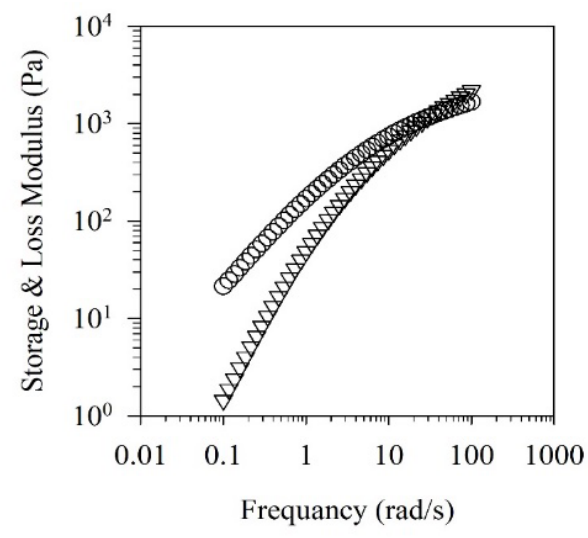

(b)

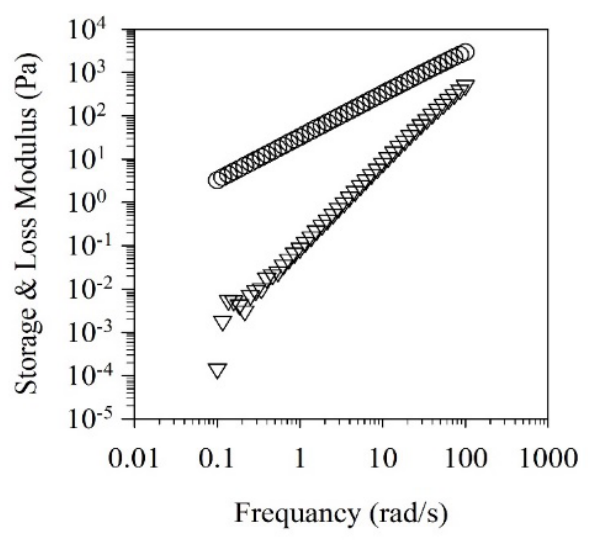

(d)

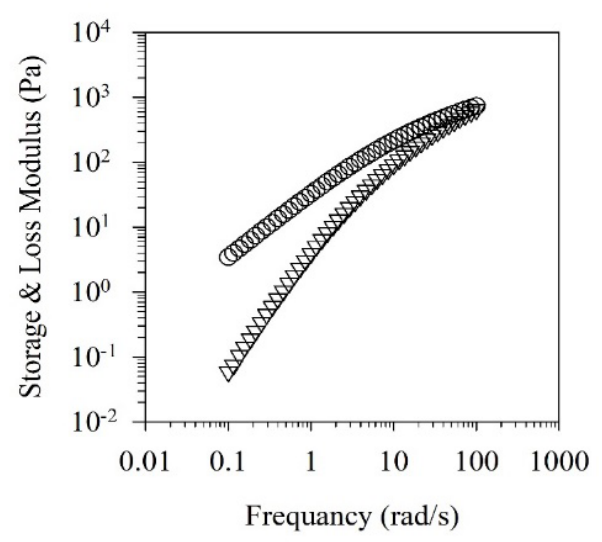

Figure 3. Frequency sweep test: the storage and loss moduli versus frequency for pure components. (a) pure PBD, (b) PDMS, (c) 5\% Boger fluid, (d) 7.5\% Boger fluid, and (e) 10\% Boger fluid.

Fig. 3 shows the results of small amplitude oscillatory shear tests (SAOS) for the pure components and Boger fluids. Those tests are performed using constant strain equal to $20 \%$ (the value was determined from the strain sweep tests) and the frequency is swept from 0.1 to $100 \mathrm{rad} / \mathrm{s}$. In parts (a), (b), and (c), which correspond to pure 
PBD, PDMS, and 5\% Boger fluid, respectively, the loss modulus (liquid-like behaviour) is larger than the storage modulus (solid-like behaviour). In part (d), which is the plot of $7.5 \%$ Boger fluid, the loss modulus is approximately equal to the storage modulus at the high-end of the frequency values. For part (e), which is $10 \%$ Boger fluid, the storage modulus values exceed the values of the loss modulus at a frequency value equals to 30 $\mathrm{rad} / \mathrm{s}$. By comparing storage and loss moduli for all the Boger fluids in the figure, the higher the percent of the high molecular weight PBD in the solution is, the higher the values of storage and loss modulus values are.

\section{CHARACTERIZATION OF POLYMER BLENDS}

\section{Interfacial Tension and Droplet Size Estimation}
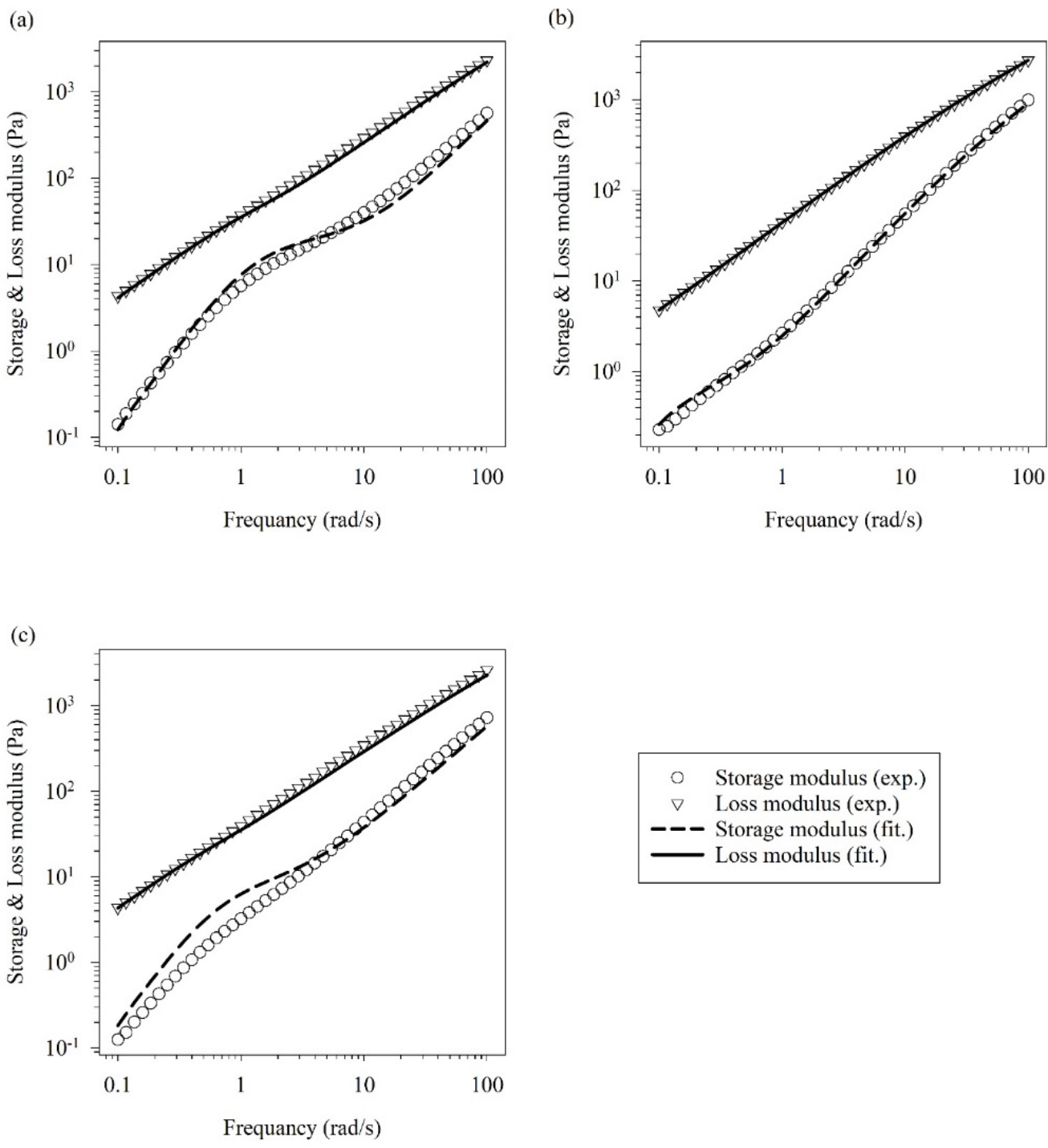

Figure 4. Comparison between the experimental storage and loss moduli of the blends and those obtained using Palierne model. (a) for BL_B0.05, (b) for BL_B0.075, and (c) for BL_B0.10. 
As can be seen in Table 2, three blends were prepared from Boger fluids. The blend of the pure PBD with PDMS is not reported because the viscosity ratio of the droplet phase to the matrix phase is very small (almost equal to 0.025 ) which is going to result in unstable droplets, and a significant droplet breakup and coalescence will occur. The interfacial tension $(\Gamma)$ values between the droplet phase and the matrix phase for all the blends that are reported in Table 2 were calculated based on Broseta et al. (1990) formula.

For the experimental investigation and modelling of LAOS, the droplet size of the blends needs to be estimated. For estimating the droplet size, Palierne model (Palierne, 1990) was used. The calculated storage and loss moduli based on the obtained droplet sizes are presented in Fig. 4. The obtained droplet size values with their standard deviation are summarized in Table 2 .

\section{LAOS of the Blends}

In this section, the LOAS tests are reported for the blends composed of the different types of Boger fluids $5.0 \%, 7.5 \%$, and $10 \%$ high molecular weight after the four-hour pre-shear period. For the LAOS tests, the frequency was set to $1 \mathrm{rad} / \mathrm{s}$, and the experiments were performed using strain amplitude ranging from $50 \%$ to $1600 \%$. In the coming graphs, different maximum strain amplitude values are reported for the blends because of significant change in droplets microstructures. For the BL_B0.050, maximum strain amplitude, equal to 300\%, is reported. For the BL_B0.075, strain amplitude up to $1000 \%$ is reported. And for the BL_B0.1, strain amplitude up to $1600 \%$ is reported. The experimental data of LAOS was directly transformed from time domain to frequency domain using the Fourier transform (FT) tool of TRIOS software of TA Instrument.

In Fig.5(a), the first harmonic versus strain amplitude for the various blends is reported. In this log-log plot for all the blends, the first harmonic is linearly increasing as the percent of the applied strain amplitude increased. And the amount of the high molecular weight used to prepare the Boger fluids is reflected on the increase in the values of the first harmonic. As the percent of the high molecular weight PBD increased, the first harmonic increased.

In Fig. 5(b), the appearance of the nonlinear stress contribution as a third harmonic can be observed. The third harmonic is increasing monotonically with the increasing strain amplitude for the BL_B0.075 and the BL_B0.1. While for the BL_B0.050, the third harmonic starts to increase with strain amplitude until it reaches its maximum at $200 \%$ strain amplitude and then starts to decrease. For strain amplitudes up to $225 \%$, the BL_B0.050 has a higher third harmonic contribution compared to the BL_B0.1 and the BL_B0.075. For the strain amplitude between 225\% and 300\%, the BL_B0.1 has a higher third harmonic contribution than the BL_B0.050 and at last the BL_B0.075. For strain amplitudes higher than $300 \%$ the BL_B0.1 has a higher third harmonic contribution than the BL_B0.075.

In Fig. 5(c), the non-linearity using the nonlinear quantitative coefficient is investigated. $Q^{3}$ is the relative intensity of the third harmonic to the first harmonic divided by the squared strain (Kyu Hyun \& Wilhelm, 2009; Reinheimer et al., 2011). For all the blends, the relative intensity shows a maximum at $100 \%$ strain amplitude. And for the all the blends, the relative intensity decreases monotonically with the increasing strain. Even though the relation between the viscosity ratio and the droplet size is increasing with increasing the amount of the higher molecular weight contents as can be seen in Table 2, the BL_B0.05 shows a higher relative intensity than the BL_B0.1, until it reaches $250 \%$ strain amplitude where the BL_B0.1 shows a higher $Q^{3}$ value than the BL_B0.050. The BL_B0.075, which has a viscosity ratio almost equal to unity, shows a lower $Q^{3}$, than the BL_B0.05 and the BL_B0.1.

To investigate this behavior, the elastocapillary number (Ec) was calculated as shown below (Li \& Sundararaj, 2010): 


$$
E c=\frac{\lambda_{1} \Gamma}{\eta_{m} R_{d}}
$$

where $\eta_{m}$ is the viscosity of the matrix phase.

The elastocapillary number is a ratio of the Deborah number to the capillary number. The higher the elastocapillary number is, the higher the elasticity contribution is. From the calculated values in Table 2, the BL_B0.050 has a higher elastic contribution compared to the BL_B0.075. On the other hand, the BL_B0.1 has the highest elastic contribution to BL_B0.050 and BL_B0.075. Therefore, this reveals the dependence of the nonlinearity on many factors including the relaxation time, viscosity, interfacial tension, droplets size, and may also include the droplet size distribution.

(a)

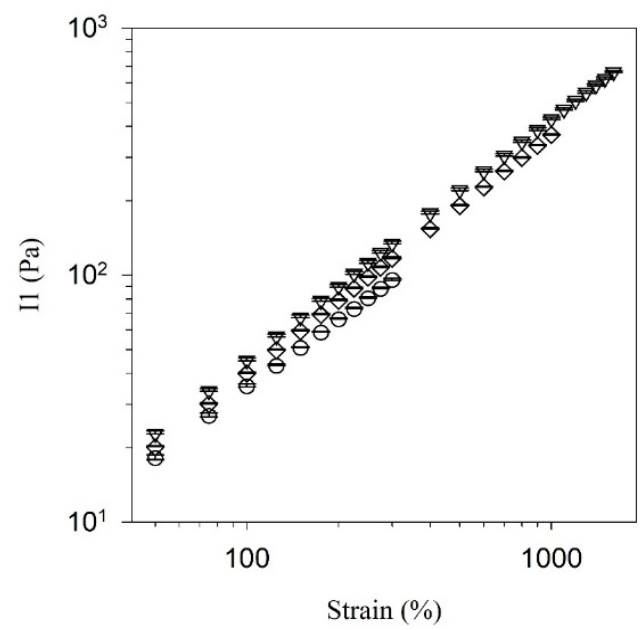

(c)

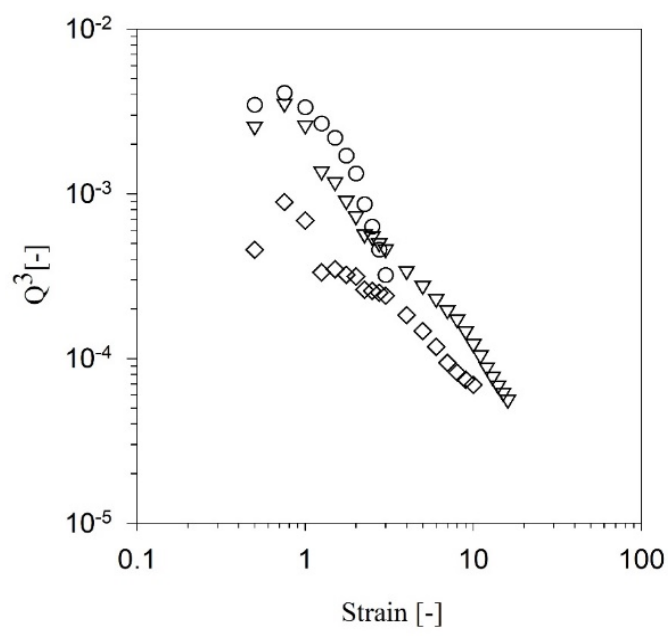

(b)

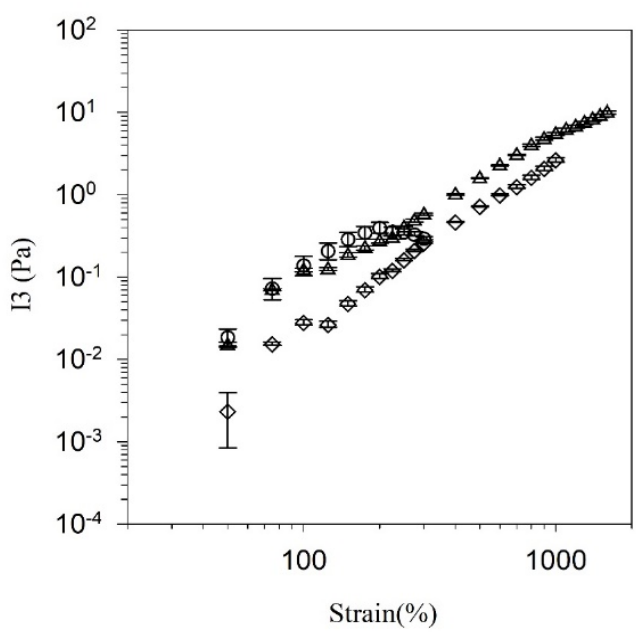

Figure 5. The experimental LAOS data for the blends: (a) the first harmonic data, (b) third harmonic data, and (c) the nonlinear quantitative coefficient. 
Table 2. The interfacial tension, viscosity ratio, the average droplets size for the blends, and the elasto-capillary number $(\mathrm{Ec})$ of the investigated blends.

\begin{tabular}{|c|c|c|c|c|c|}
\hline Designation & Type of blend & $\boldsymbol{\Gamma}(\mathbf{m N} / \mathbf{m})$ & $\boldsymbol{\eta}_{\mathbf{r}}$ & Average droplets size $(\boldsymbol{\mu m})$ & Ec \\
\hline BL_B0.050 & $\begin{array}{c}80 \% \text { PDMS with 20\% of } \\
\text { the 5.0\% Boger fluid. }\end{array}$ & 2.59 & 0.319 & $36.33 \pm 1.42$ & 0.0785 \\
\hline BL_B0.075 & $\begin{array}{c}80 \% \text { PDMS with 20\% of } \\
\text { the 7.5\% Boger fluid. }\end{array}$ & 2.61 & 0.967 & $43.85 \pm 4.53$ & 0.0760 \\
\hline BL_B0.10 & $\begin{array}{c}80 \% \text { PDMS with 20\% of } \\
\text { the 10\% Boger fluid. }\end{array}$ & 2.65 & 6.227 & $91.84 \pm 15.03$ & 0.1623 \\
\hline
\end{tabular}

\section{Modeling the polymer blends with CV-model}

(a)

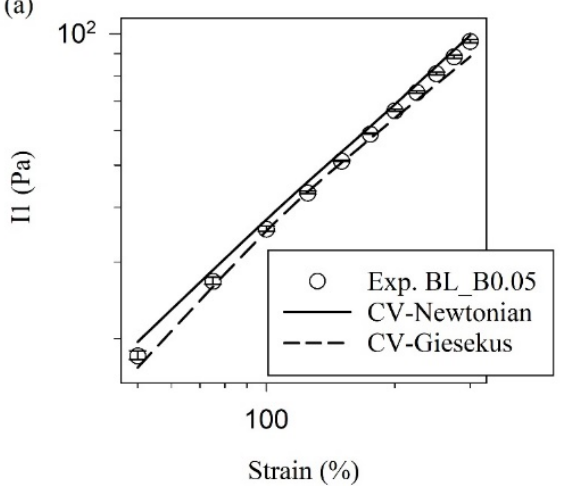

(c)

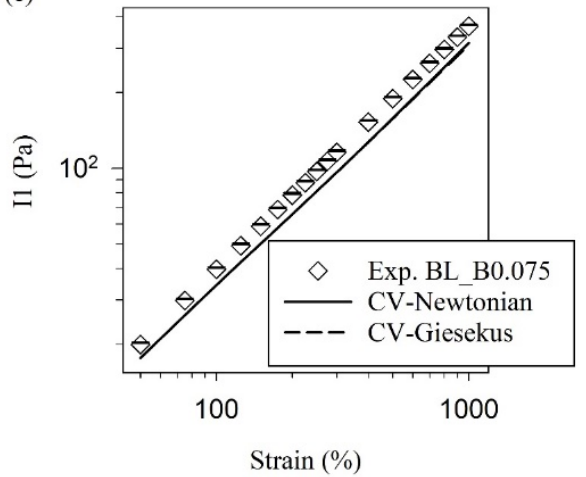

(b)

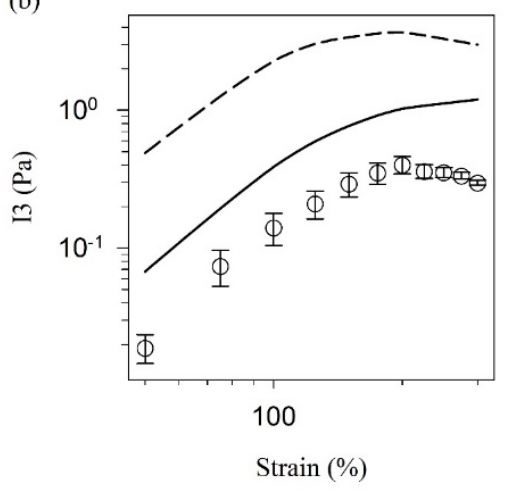

(d)

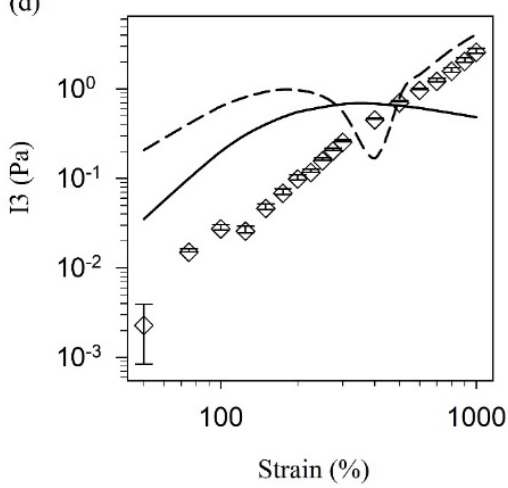



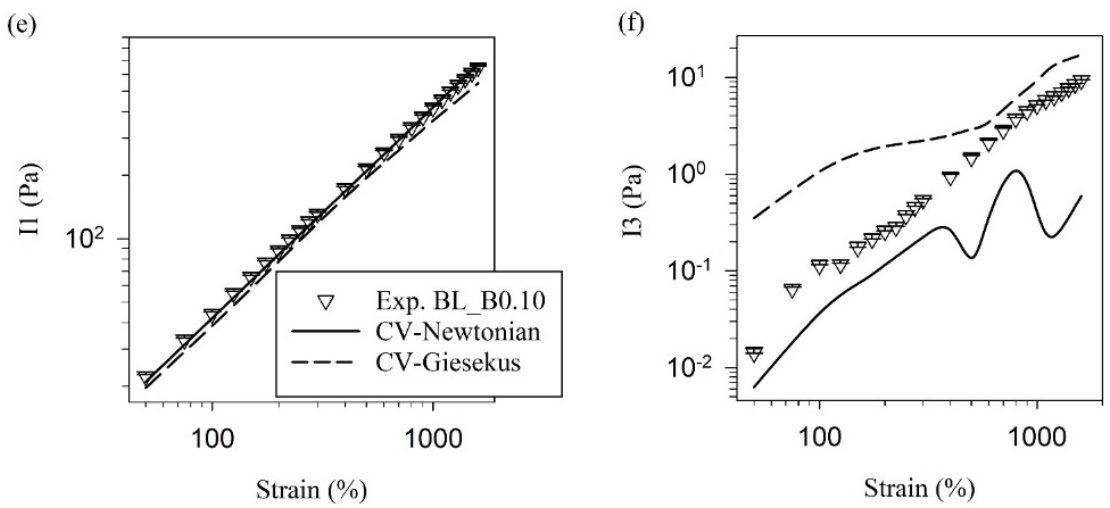

Figure 6. The experimental stress harmonics compared to the prediction of the Newtonian CV-model and the non-Newtonian CV-model.

For the Newtonian CV-model, the non-linear behavior is a result of the interfacial forces between the droplet phase and the matrix phase. For the modified non-Newtonian CV-model, the non-linearity behavior results from the interfacial forces and from the non-Newtonian character of the droplet phase. Therefore, in this part of the FTLAOS, experimental results are compared with the numerical results obtained from the Newtonian CV-model and the modified non-Newtonian CV-model. Both CV models are written on Mathematica software where the coupled ordinary differential equations are solved by the fourth order Runge-Kutta method. The final obtained stresses from the constitutive equations are functions of time and were transformed to the frequency domain by using Mathematica Fourier transform function.

In Figures 6(a), (b), and (c) for the BL_B0.05, the CV-Newtonian and the non-Newtonian (CV-Giesekus) models are capturing the first harmonic with slight difference between the two models. For the third harmonic in Fig. 6(b), for BL-B0.05, both the CV-Newtonian model and the CV-Giesekus are capturing the behavior qualitatively. But the CV-Newtonian is quantitatively better than the CV-Giesekus. For BL_B0.075 Fig. 6(d), CVNewtonian is showing better behavior than CV-Giesekus for strain up to $400 \%$, while at higher strain the CVNewtonian totally failed possibly due to the shear thinning behavior at this range (the shear rate is equal to $4 \mathrm{~s}^{-1}$ ), and, at this range, the behavior is captured by CV-Giesekus. For BL_B0.10, Fig. 6(f), the CV-Newtonian model is also better than CV-Giesekus quantitatively at strain values lower than $400 \%$. At higher strain, the CV-Giesekus captures the behavior qualitatively and quantitatively, while the CV-Newtonian failed to capture it.

\section{CONCLUSIONS}

Under LAOS conditions and using Fourier transform (FT) as analysis technique, experimental and phenomenological modeling investigations were carried out for the case of immiscible polymer blends composed of non-Newtonian (Boger fluids) droplet phase and a Newtonian matrix phase. In the experimental FT-LAOS part, the results were analyzed based on $Q^{3}$, which is the relative intensity of the third harmonic to the first harmonic divided by the squared strain amplitude, and, for all blends, $Q^{3}$ was decreasing monotonically with increasing strain amplitude. The blend composed of the Boger fluid of $7.5 \%$ high molecular weight, which has a viscosity ratio equal to unity, has the lowest $Q^{3}$ values compared to the other types of blends that have a viscosity ratio lower than unity and higher than unity. And this due to the dependence of the non-linearity on various factors such as the viscosity ratio, relaxation time, interfacial tension, and the droplets size. 
The CV-model that accounts for the non-Newtonian droplet phase was modified by creating a representative viscosity that accounts for the character of Boger fluid behavior. In addition, for the blend stress calculations, the droplet stress contribution was represented by Giesekus constitutive equation. The proposed model was tested and compared with the experimental FT-LAOS. From the overall results, the CV-Newtonian model captures the behavior at low strain better than the CV-Giesekus, while, at high strain values, the CV-Giesekus was capturing the behavior better than the CV-Newtonian model for the viscosity ratio, which is equal to unity and larger than unity values.

\section{ACKNOWLEDGEMENTS}

The authors express their gratitude to Nanotechnology Research Facility at Kuwait University (Project no. GE01/07) for allowing them to use ARES-G2 rheometer.

\section{REFERENCES}

Aggarwal, N., \& Sarkar, K. 2007. Deformation and breakup of a viscoelastic drop in a Newtonian matrix under steady shear. Journal of Fluid Mechanics, 584, 1-21.

Almusallam, A. S. 2013. The modified constrained volume model predictions in shearing flow at nonunity viscosity ratio values. Rheologica Acta, 52(6), 607-621.

Almusallam, A. S. 2014. Large Amplitude Oscillatory Shear of Immiscible Polymer Blends and Comparison to Anisotropy and Droplet Models. Journal of Rheology, 58(6), 1903-1916.

Almusallam, A. S., \& Bini, T. B. 2018. Scaling Law Accomplished through Correlation of Large Amplitude Oscillatory Shear of Immiscible Polymer Blends with Jackson and Tucker Model. Applied Rheology, 28(4).

Bird, R. B., \& Hassager, O. 1987. Dynamics of Polymeric Liquids: Fluid mechanics: Wiley.

Broseta, D., Fredrickson, G. H., Helfand, E., \& Leibler, L. 1990. Molecular weight and polydispersity effects at polymer-polymer interfaces. Macromolecules, 23, 132-139.

Carotenuto, C., Grosso, M., \& Maffettone, P. L. 2008. Fourier Transform Rheology of Dilute Immiscible Polymer Blends: A Novel Procedure To Probe Blend Morphology. Macromolecules, 41, 4492-4500.

Cheng, J., \& Manas-Zloczower, I. 1990. Flow field characterization in a banbury mixer. International Polymer Processing, 5(3), 178-183.

Debbaut, B., \& Crochet, M. J. 1988. Extensional effects in complex flows. Journal of Non-Newtonian Fluid Mechanics, 30(2), 169-184.

Deyrail, Y., Huneault, M. A., \& Bousmina, M. 2009. Rheo-optical study of nonlinear effects in an immiscible newtonian polymer blend under large amplitude oscillatory shear flow. Journal of Polymer Science, Part B: Polymer Physics, 47(15), 1467-1480.

Doi, M., \& Ohta, T. 1991. Dynamics and rheology of complex interfaces. I. The Journal of Chemical Physics, 95(2), 1242-1248.

Filipe, S., Cidade, M. T., Wilhelm, M., \& Maia, J. M. 2006. Evolution of the morphological and rheological properties along the extruder length for compatibilized blends of a commercial liquid-crystalline polymer and polypropylene. Journal of Applied Polymer Science, 99(1), 347-359.

Grosso, M., \& Maffettone, P. L. 2007. A new methodology for the estimation of drop size distributions of dilute polymer blends based on LAOS flows. Journal of Non-Newtonian Fluid Mechanics, 143, 48-58. 
Hyun, K., \& Wilhelm, M. 2009. Establishing a New Mechanical Nonlinear Coefficient Q from FT-Rheology: First Investigation of Entangled Linear and Comb Polymer Model Systems. Macromolecules, 42(1), 411422.

Hyun, K., Wilhelm, M., Klein, C. O., Cho, K. S., Nam, J. G., Ahn, K. H., . . McKinley, G. H. 2011. A review of nonlinear oscillatory shear tests: Analysis and application of large amplitude oscillatory shear (LAOS). Progress in Polymer Science, 36, 1697-1753.

Jackson, N. E., \& Tucker, C. L. 2003. A model for large deformation of an ellipsoidal droplet with interfacial tension. Journal of Rheology, 47, 659-682.

Lerdwijitjarud, W., Larson, R. G., Sirivat, A., \& Solomon, M. J. 2003. Influence of weak elasticity of dispersed phase on droplet behavior in sheared polybutadiene/poly(dimethyl siloxane) blends. Journal of Rheology, 47(1), 37-58.

Li, H., \& Sundararaj, U. 2010. Experimental investigation of viscoelastic drop deformation in Newtonian matrix at high capillary number under simple shear flow. Journal of Non-Newtonian Fluid Mechanics, 165(19-20), 1219-1227.

Maffettone, P. L., \& Minale, M. 1998. Equation of change for ellipsoidal drops in viscous flow. Journal of Non-Newtonian Fluid Mechanics, 78, 227-241.

Minale, M. 2010. Models for the deformation of a single ellipsoidal drop: a review. Rheologica Acta, 49, 789806.

Ock, H. G., Ahn, K. H., Lee, S. J., \& Hyun, K. 2016. Characterization of Compatibilizing Effect of Organoclay in Poly(lactic acid) and Natural Rubber Blends by FT-Rheology. Macromolecules, 49(7), 2832-2842.

Palierne, J. F. 1990. Linear rheology of viscoelastic emulsions with interfacial tension. Rheologica Acta, 29(3), 204-214.

Peters, G. W. M., Hansen, S., \& Meijer, M. E. H. 2001.Constitutive modeling of dispersive mixtures. Journal of Rheology, 45, 659-689.

Reinheimer, K., Grosso, M., \& Wilhelm, M. 2011. Fourier Transform Rheology as a universal non-linear mechanical characterization of droplet size and interfacial tension of dilute monodisperse emulsions. Journal of Colloid and Interface Science, 360, 818-825.

Salehiyan, R., Choi, W. J., Lee, J. H., \& Hyun, K. 2014. Effects of mixing protocol and mixing time on viscoelasticity of compatibilized PP/PS blends. Korea Australia Rheology Journal, 26(3), 311-318.

Salehiyan, R., \& Hyun, K. 2013. Effect of organoclay on non-linear rheological properties of poly(lactic acid)/poly(caprolactone) blends. Korean Journal of Chemical Engineering, 30(5), 1013-1022.

Salehiyan, R., Song, H. Y., Choi, W. J., \& Hyun, K. 2015. Characterization of Effects of Silica Nanoparticles on (80/20) PP/PS Blends via Nonlinear Rheological Properties from Fourier Transform Rheology. Macromolecules, 48(13), 4669-4679.

Salehiyan, R., Song, H. Y., Kim, M., Choi, W. J., \& Hyun, K. 2016.Morphological Evaluation of PP/PS Blends Filled with Different Types of Clays by Nonlinear Rheological Analysis. Macromolecules, 49(8), 3148-3160.

Salehiyan, R., Yoo, Y., Choi, W. J., \& Hyun, K. 2014. Characterization of morphologies of compatibilized Polypropylene/ Polystyrene blends with nanoparticles via nonlinear rheological properties from FTrheology. Macromolecules, 47(12), 4066-4076.

Verhulst, K., Moldenaers, P., \& Minale, M. 2007. Drop shape dynamics of a Newtonian drop in a nonNewtonian matrix during transient and steady shear flow. Journal of Rheology, 51(2), 261-273. 
Yu, W., \& Bousmina, M. 2003. Ellipsoidal model for droplet deformation in emulsions. Journal of Rheology, 47(4), 1011-1039.

Yu, W., Bousmina, M., Grmela, M., \& Zhou, C. 2002. Modeling of oscillatory shear flow of emulsions under small and large deformation fields. Journal of Rheology, 46(6), 1401-1418.

Yu, W., \& Zhou, C. 2007. A simple constitutive equation for immiscible blends. Journal of Rheology, 51(2), 179-194. 